\title{
Conventional and Molecular Studies of Brown Planthopper (Nilaparvata lugens Stal) Resistance Genes in Rice: A Basis for Future Study of Natural Insect Resistance Genes Using Molecular Markers in Nepal
}

\author{
Prem N. Sharma ${ }^{1}$, Naoki Mori ${ }^{2}$, Shigeo Takumi ${ }^{2}$ and Chiharu Nakamura ${ }^{2}$ \\ ${ }^{1}$ Entomology Division, Nepal Agricultural Research Council, Lalitpur, Nepal \\ ${ }^{2}$ Faculty of Agriculture, Kobe University, Kobe, Japan \\ e-mail: premnidhi@yahoo.com
}

\begin{abstract}
Rice productivity is greatly affected by various biotic and abiotic stresses. Insect-pests are one of the major biotic constraints to cause significant losses in rice production. Brown planthopper (BPH), Nilaparvata lugens Stål, is the most serious insect-pest of rice in Asia where most of the world rice is produced. Controlling insects using chemicals is already proven detrimental not only to environment but also to human health. Integrated Pest Management (IPM) is the best approach to control insect pests. Host plant resistance is the principal component of IPM along with biological, cultural and physical methods. Use of varietal resistance is the best option to control BPH. Many BPH resistant rice varieties with natural BPH resistance have been developed and widely used against $\mathrm{BPH}$. However, frequent breakdown of monogenic resistance by new BPH biotypes has been a serious threat to control BPH. To overcome such difficulty in the use of monogenic resistance, development of durable resistance is needed as the sustainable means to control BPH. To develop durable resistance, pyramiding of BPH resistance genes and quantitative trait loci (QTLs), through marker-assisted method, is needed. For this, many BPH resistance genes and QTLs have already been identified and mapped on rice chromosomes. This article reviews identification, mapping and pyramiding toward successful cloning of BPH resistance genes/QTLs and provides the basis/ guidelines to work on natural insect resistance genes using molecular markers in Nepal.
\end{abstract}

Key words: rice, brown planthopper (BPH), Nilaparvata lugens, resistance genes, QTLs, mapping, pyramiding

\section{Introduction}

Rice is the most important staple food crop of the world, and Asia is the main region of its cultivation. Rice production, however, has been threatened by insectpests as one of the major biotic constraints. The brown plant hopper (BPH), Nilaparvata lugens Stal (Homoptera: Delphacidae) is one of the most destructive insect-pests of rice in Asia and is distributed worldwide where the rice is cultivated. Frequent outbreaks of BPH have been reported particularly in tropical, subtropical and temperate regions in South, Southeast and East Asia and Oceania. $\mathrm{BPH}$ damages rice plants directly by feeding the phloem sap as a result rice plants show severe damage symptoms commonly referred to hopper-burn. BPH also damages rice plants indirectly by acting as a vector in transmitting viral diseases such as grassy stunt and ragged stunt. Both direct and indirect damages by $\mathrm{BPH}$ cause considerable yield loss of rice in Asia (Rivera et al. 1966, Ling et al. 1978, Sogawa \& Cheng 1979).

The availability of broad-spectrum insecticides together with chemical fertilizers and improved crop varieties, after the Green Revolution, greatly led the farmers to depend on agro-chemicals for controlling insects including the BPH. However, excessive reliance 
on agro-chemicals including insecticides has resulted in adverse side effects such as development of pesticide resistance in insect pests, pest resurgence, elimination of beneficial insects including natural enemies and serious environmental pollution along with human health hazards (Georghiou 1986, Chelliah \& Heinrich 1980, Heinrichs et al. 1982). Many BPH and white-backed planthopper (WBPH) outbreaks were reported in farmers' fields to be induced by insecticides (Heinrichs et al. 1982, Salim \& Heinrichs 1987). To overcome such negative impacts in the use of chemical pesticides, scientists developed an ecological approach for pest control, which is now popularly known as integrated pest management (IPM) (Huffaker \& Smith 1980). IPM utilizes host plant resistance as a principle component along with cultural and biological methods together with judicious use of agro-chemicals. The host plant resistance i.e. the natural resistance that exists in host plant species and their relatives has been challenged for long enough time during evolution should provide promising and more readily acceptable means to control insect pests. To effectively utilize such natural resistance systems in IPM, the resistance mechanism should be fully understood at molecular level. For this, natural insect resistance genes have been cloned and a number of studies have been conducted for elucidation of their structure, expression and function (Sharma et al. 2003a).

Here in this article, towards map-based cloning of $\mathrm{BPH}$ resistance genes as a model case, identification, mapping, cloning and pyramiding of BPH resistance genes/QTLs are reviewed; and future strategy to develop rice varieties with durable resistance against virulent $\mathrm{BPH}$ biotypes has been discussed as well. In addition, future prospect and guidelines for studying varietal resistance against BPH using molecular markers in Nepal are illustrated.

\section{Use of varietal resistance against BPH and resistance breakdown by new BPH biotypes in Asia}

Development of efficient mass-screening techniques such as bulked seedling test and mass-rearing methods of BPH (Athwal et al. 1971, Kaneda \& Kisimoto 1979, Choi et al. 1979) accelerated the work on varietal resistance against BPH in Asian countries especially at the International Rice Research Institute (IRRI) and Japan. Thousands of world germ plasm collections were screened and large numbers of resistance sources identified as well as many BPH resistant varieties were bred and cultivated in the Philippines, Japan and other Asian countries since 1970s. Unfortunately, during 1970s, continuous and intensive cultivation of resistant rice varieties in some tropical countries of Asia created a serious problem in BPH control due to the frequent appearance of new and virulent BPH biotypes. The first BPH resistant cultivar IR26 with $\mathrm{Bphl}$ gene was released in the Philippines in 1973 and in Indonesia and Vietnam in 1974; and it was widely grown in these countries. But its genetic resistance was broken down in the Philippines in 1977 and in Indonesia and Vietnam in 1978 due to the occurrence of new BPH biotypes (Feuer 1976, Anonymous 1975, Stapley 1975). Another $\mathrm{BPH}$ resistant cultivar IR36 bred to carry bph2 gene was also broken down by more virulent BPH biotype (IRRI 1982, Murata et al. 1998). Thus, breakdown of $\mathrm{BPH}$ resistance one after another due to the frequently developed BPH biotypes created serious difficulty in breeding for host plant resistance against BPH. It was therefore necessary to look for additional genetic sources for widening the genetic base to develop the durable BPH control strategy. The occurrence of new BPH biotypes compelled the scientists to search for additional sources of resistance to cope with such frequently developed virulent BPH biotypes.

\section{Genetic analysis and identification of BPH resistance genes in rice}

The work on rice resistance against BPH was undertaken only after the establishment of IRRI in the Philippines in 1960 which initiated the studies on varietal resistance to this pest in 1966 . The breeding programs to develop BPH-resistant rice varieties were initiated in 1968. Athwal et al. (1971) initially crossed four resistant varieties, Mudgo, C022 and MTU15 (all Bphl gene donors) and ASD7 (a $b p h 2$ gene donor) with a susceptible TN1, and identified two loci for resistance, $B p h 1$ and $b p h 2$. Using the biotype 1 as the test insect, Lakshmi narayana \& Khush (1977) analyzed 28 varieties and identified two new genes, Bph3 and $b p h 4$. The two Sri Lankan varieties, Rathu Heenati and Babawee were the donors for Bph3 and bph4 respectively. Another new gene $b p h 5$ was identified when the crosses were made between ARC10550 and TN1 by using the test insect biotype 4 . ARC 10550 was resistant to biotype 4 in South Asia but it is susceptible to all the three biotypes, biotype 1,2 and 3 in Southeast Asia (Khush et al. 1985). Kabir \& Khush (1988) 
Prem N. Sharma et al./Conventional and Molecular Studies .......

analyzed additional 17 rice varieties resistant to biotype 4 but susceptible to biotype 1, 2, and 3 using biotype 4 as the test insects, and identified two new genes, Bph6 and bph7. The genes Bph6 and $b p h 7$ were carried by Swarnalata and T12 respectively. Nemoto et al. (1989) identified a new gene bph8 in three varieties, TC5 (Thai collection 5), TC11, and Chin Saba. This gene was allelic in these varieties and conferred resistance to biotypes 1,2 , and 3 . Ikeda (1985) identified a new gene Bph9 in three varieties, Kaharamana, Balamawee and Pokkali. This gene was also allelic in these varieties but was different from $B p h l$ and $B p h 3 . B p h 10$ was the first gene identified in a wild relative (Oryza australiensis) of rice (Jena \& Khush 1990) which was later introgressed into an indica breeding line (Ishii et al. 1994). Other two BPH resistance genes identified in a wild species (Oryza officinalis) of rice were $b p h 11(\mathrm{t})$ and $b p h 12(\mathrm{t})$ (Hirabayashi et al. 1998, 1999). Besides these major genes, several major/minor QTLs of BPH resistance have been identified in rice and its wild relatives. QTLs for BPH resistance were first identified by Alam et al. (1998) using the doubled haploid lines (DHLs) derived from a cross between an indica variety IR64 and a japonica variety Azucena using the BPH populations collected from IRRI farm (IRRI colony) and Central Luzon Island (CL colony) in the Philippines. These colonies were fully adapted to Bph1. IR64 showed moderate resistance response to these populations. This study detected seven significant QTLs that were scattered over on six different rice chromosomes (Khush 1989, Cohen 1997, Sharma et al. 2003a). Other two major BPH resistance QTLs, $Q b p 1$ and $Q b p 2$, were identified by Chinese researchers from the crosses between B5, a BPH resistant breeding line with introgressed resistance genes from $O$. officinalis, and Minghui 63, a susceptible indica cultivar, using the BPH biotypes 1 and 2 (Huang et al. 2001, Sharma et al. 2003a). Xu et al. (2002) further identified seven main effect QTLs and many epistatic QTLs for quantitative resistance to $\mathrm{BPH}$ using the recombinant inbred lines (RILs) derived from a Lemont and Teqing cross. Lemont is moderately resistant and Teqing is highly susceptible to BPH. The BPH biotypes were thus found always useful in resistance breeding against $\mathrm{BPH}$ in rice. By now, at least 24 major genes and several major/minor QTLs against BPH have been identified in rice cultivars and its wild relatives. Out of the 24 major resistance genes, at least 19 genes have already been mapped on rice chromosomes (Sharma et al. 2003a, Jena et al. 2006, Chen et al. 2006, Jairin et al. 2007, Rahman et al. 2009, Brar et al. 2009, Myint et al. 2012). Twelve major genes including the major/minor QTLs of BPH resistance initially identified and mentioned in this section have been shown in Table 1 .

Table 1. List of initially identified and/or mapped brown planthopper resistance genes/QTLs

\begin{tabular}{|c|c|c|c|c|}
\hline Gene & $\begin{array}{l}\text { Year of } \\
\text { identification }\end{array}$ & $\begin{array}{l}\text { Resistance to } \\
\text { biotypes }\end{array}$ & Source & Chromosome \\
\hline Bphl & 1971 & 1,3 & Mludgo & 12 \\
\hline bph2 & 1971 & 1,2 & ASD7, IR1154-243 & 12 \\
\hline$B p h 3$ & 1977 & $1,2,3,4$ & Rathu Heenati & \\
\hline bph4 & 1977 & $1,2,3,4$ & Babawee & 6 \\
\hline bph 5 & 1985 & 4 & ARC10550 & \\
\hline$B p h 6$ & 1988 & 4 & Swamalata & \\
\hline bph? & 1988 & 4 & T12 & \\
\hline bph 8 & 1989 & $1,2,3$ & Thai collection 5, 11 and Chin Saba & \\
\hline$B p h 9$ & 1985 & $1,2,3$ & Pokkali, Balamawee and Kaharanama & 12 \\
\hline Bph10 & 1994 & $1,2,3$ & & 12 \\
\hline bphI $\mathrm{I}(\mathrm{t})$ & 1998 & & & 3 \\
\hline$b p h i 2(\mathrm{t})$ & 1999 & & & 4 \\
\hline QTIs & 1998 & & & $1,2,3,4,6,8$ \\
\hline QTIs & 2002 & & & $1,3,5,8,11$ \\
\hline Obpl & 2001 & & & \\
\hline$O b p 2$ & 2001 & & & 4 \\
\hline
\end{tabular}




\section{Chromosomal assignment, mapping, pyramiding toward cloning of major genes/ QTLs of BPH resistance by molecular marker assisted method}

Of the 12 major BPH resistance genes mentioned above, at least seven of them have already been assigned to and mapped on individual rice chromosomes (Table 1). The chromosomal assignment and determination of precise position of $\mathrm{BPH}$ resistance genes on rice chromosomes have accurately been achieved only when the molecular marker based studies started since 1990s. Initially, Bph1 and bph2 were assigned to chromosome 4; and Bph3 and bph4 to chromosome 10 by classic trisomic analysis and linkage analysis using morphological markers (Ikeda \& Kaneda 1983, Ikeda 1985). However, using markerassisted selection (MAS) method, chromosomal assignment of these genes was later corrected. Bphl was mapped in a breeding line TKM6 on rice chromosome 12 by RFLP (restriction fragment length polymorphism)-marker based analysis (Hirabayashi \& Ogawa 1995) and this study was later confirmed by RAPD (random amplified polymorphic DNA) and RFLP-markers based mapping studies using different mapping populations (Murata et al. 1997, Murata et al. 1998 and Jeon et al. 1999). The gene bph2, which was previously considered recessive in indica background was confirmed to be dominant in japonica background and mapped on the long arm of chromosome 12 close to the dominant gene Bphl based on RFLP and AFLP (amplified fragment length polymorphism) analyses using large mapping populations (Murai et al. 2001, Sharma et al. 2003b). Based on bulked segregant and linkage analyses using RFLP markers, the $b p h 4$ gene from the Sri Lankan indica variety Babawee was reassigned to the distal region of the short arm of chromosome 6 instead of chromosome 10 (Michelmore et al. 1991, Kawaguchi et al. 2001). Based on RFLP marker analysis, a dominant gene $B p h 10$ in an indica line, introgressed from $O$. australiensis, was mapped on the long arm of chromosome 12 and another dominant gene Bph9 from a Sri Lankan variety Pokkali was also mapped on the long arm of chromosome 12 (Ishii et al. 1994, Murata et al. 2001). Besides mapping of several major genes of BPH resistance, many major/minor QTLs of BPH resistance have also been mapped on rice chromosomes. Alam et al. (1998) were the first who detected seven significant QTLs for BPH resistance in a moderately resistant variety IR64; the QTLs were located on 6 of the 12 rice chromosomes (Khush 1989, Cohen et al. 1997). Two major BPH resistance QTLs, Qbp1 and $Q b p 2$, were further identified by Chinese researchers in a BPH resistant breeding line B5 possessing resistance genes from $O$. officinalis (Huang et al. 2001). Five and six RFLP markers were detected from the contiguous regions of chromosome 3 and 4 , respectively, using the bulk DNA from resistant and susceptible individuals through bulked segregant analysis. A linkage analysis then mapped the two QTLs, $Q b p 1$ and $Q b p 2$ on the long arm of chromosome 3 and the short arm of chromosome 4, respectively. Since the same resistance source i.e. O. officinalis was used in the above studies, the two major genes $\operatorname{bph} 11(\mathrm{t})$ and $b p h 12(\mathrm{t})$ respectively identified on chromosome 3 and 4 in the previous studies (Hirabayashi et al. 1998, Hirabayashi et al. 1999) might represent the two major QTLs, $Q b p 1$ and $Q b p 2$, respectively (Sharma et al. 2003a). A further study (Xu et al. 2002) identified seven main effect QTLs and many epistatic QTLs for quantitative resistance to BPH in a moderately resistant indica variety Teqing. These QTLs were found distributed over all the 12 rice chromosomes.

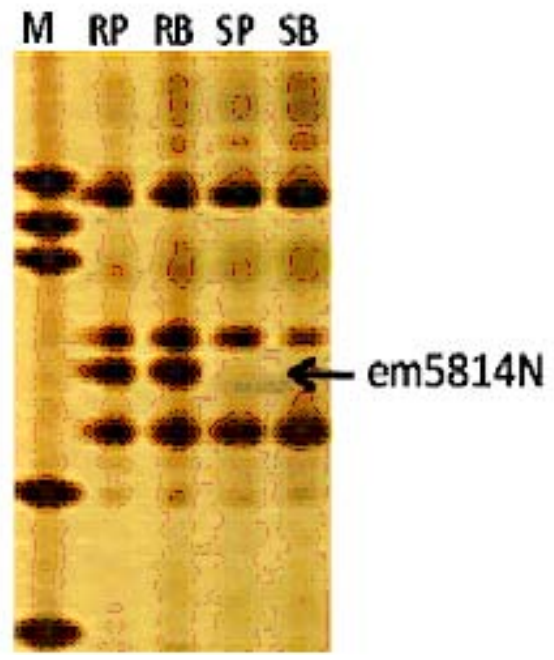

Fig. 1. A bulked segregant analysis profile of an AFLP marker on a polyacrylamide gel used to detect the Bph1 linked AFLP markers. em5814N is an AFLP marker linked to the Bphl gene. RP: DNA from resistant parent. RB: bulked DNA from resistant $\mathrm{F}_{2}$ s. SP: DNA from susceptible parent. SB: bulked DNA from susceptible $\mathrm{F}_{2} \mathrm{~s}$. M: DNA size marker (Sharma et al. 2003b) 
Prem N. Sharma et al./Conventional and Molecular Studies .......

Towards pyramiding of BPH resistance genes and map-based cloning, construction of fine linkage maps for BPH resistance genes were required. Accordingly, as a first attempt, high resolution linkage maps for Bphl and Bph2 were constructed and tightly linked molecular markers were identified for these genes. Pyramiding of these genes was also successfully achieved by marker-assisted method using large mapping populations and tightly linked molecular markers. To construct high-resolution linkage maps for Bph1 and Bph2, respectively, Sharma et al. (2003b) and Murai et al. (2001) adopted a high efficiency gene scanning system (Mano et al. 2001) that enabled efficient AFLP marker selection and mapping using a large number of segregating progenies. To fine-map Bphl, BPH bioassay and DNA analyses were performed for $\mathrm{F}_{2}$ and $\mathrm{F}_{3}$ families derived from a cross between Tsukushibare, a susceptible japonica variety as a maternal parent, and Norin-PL3, a japonica Bphlintrogression line as a paternal parent. Polymorphic AFLP markers associated to Bphl were detected through the bulked segregant analysis (Fig. 1) and were employed in the linkage analysis (Fig. 2) using the DNA of $\mathrm{F}_{2}$ segregating individuals. The Bphl locus was mapped within the region of $5.8 \mathrm{cM}$ between two molecular markers, em5814N (AFLP marker) and R2708 (RFLP marker). The two closest AFLP markers, em5814N and em $2802 \mathrm{~N}$ associated to $B p h l$ locus were located at $2.7 \mathrm{cM}$ proximal to the $B p h 1$ locus on the long arm of chromosome 12 (Sharma et al. 2003b). For constructing a high resolution linkage map for $B p h 2$, $\mathrm{F}_{4}-\mathrm{F}_{5}$ families derived from a cross between Tsukushibare and Norin-PL4 (a japonica Bph2introgression line) were subjected to BPH bioassay followed by bulked segregant and linkage analyses. The Bph2 locus was delimited with eight AFLP markers within the region of $4.2 \mathrm{cM}$ on the long arm of chromosome 12 (Murai et al 2001). A closest AFLP marker, KAM4, showed a complete co-segregation with $B p h 2$ and, the Bph2 was mapped between two AFLP markers, KAM2 and KAM3, within a 1.1-cM region (Murai et al. 2001). These AFLP markers (six markers) were successfully converted into PCR-based STS (five markers named KPMs) and CAPS (one marker named KPM2) markers (Murai et al. 2001, Sharma et al. 2004). These converted PCR-based STS (sequence tagged sites) and CAPS (codominant cleaved amplified polymorphism sequence) markers could predict that Bphl and Bph2 located at 10-cM apart on the long arm of chromosome 12 (Sharma et al. 2004). After accomplishing the construction of fine linkage maps for Bph1 and Bph2 and confirming that they were nonallelic, pyramiding of these genes was successfully achieved by Sharma et al. (2004) by marker-assisted method using the identified tightly linked markers. Briefly, for marker-assisted pyramiding of Bphl and $B p h 2$ genes, crosses were made between homozygous carrier parents for Bphl and Bph2 genes that were derived from Tsukushibare/Norin-PL3 and Tsukushibare/Norin-PL4 crosses, respectively. Tightly linked molecular markers of Bphl, one co-dominant AFLP marker (em24G) and two resistant (R)-associated dominant AFLP markers (em5814N and em32G) were used to identify the genotypes of $B p h l$ carrier lines. Similarly, tightly linked markers of Bph2, one codominant marker (KPM2) and two R-associated dominant markers (KAM4 and KPM3) were used to identify the genotypes of $B p h 2$ carrier lines. Using these molecular markers, the DNA of $\mathrm{F}_{2}, \mathrm{~F}_{3}$ and $\mathrm{F}_{4}$ progenies were analyzed for searching the useful recombinants (pyramid lines) that possessed both the genes in the homozygous condition. Two recombinant plants (rice line no. 64-4 and 64-6) were obtained in $\mathrm{F}_{3}$ progenies, both of which were reconfirmed to be the pyramided plants in $\mathrm{F}_{4}$ progenies by marker assisted selection (Sharma et al. 2004) (Fig. 3).

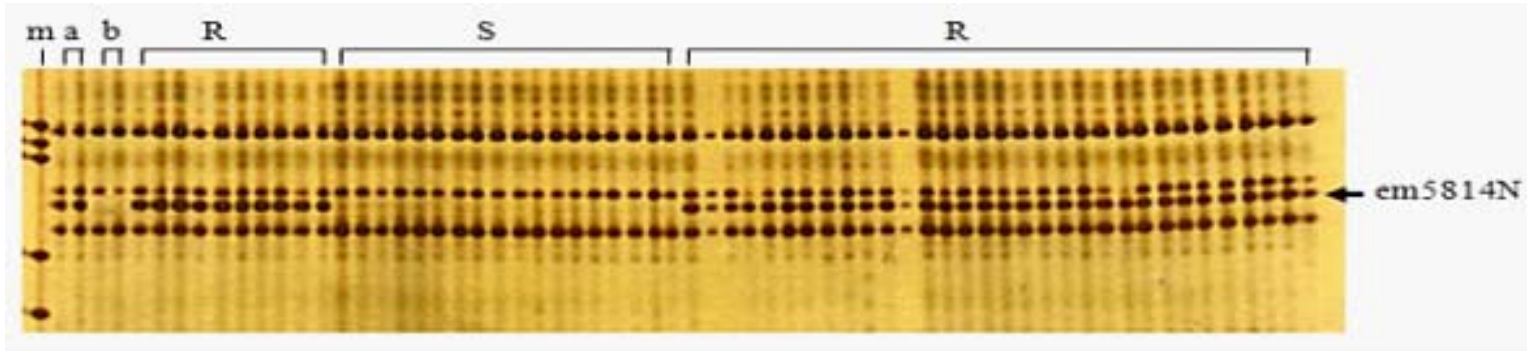

Fig. 2. A linkage analysis profile using an AFLP marker 'em5814N' associated to $B p h 1$ on a polyacrylamide gel. R \& S: resistant and susceptible lines of rice, a \& b: resistant and susceptible ckecks respectively. m: DNA size marker (Sharma et al 2003b) 
As above, a considerable progress has so far been achieved in identification, determination of carrier chromosomes and linkage mapping of BPH resistance genes/QTLs. However, no BPH resistance genes/ QTLs have been successfully cloned yet. Towards map based cloning of BPH resistance genes, construction of fine linkage maps at least for Bphl and $B p h 2$ has already been accomplished (Sharma et al. 2003b, Murai et al. 2001), and these closely linked genes have already been pyramided (Sharma et al.
2004). These genes, hopefully, will be cloned in future through map based cloning approach.

Recently, many additional major genes and QTLs of $\mathrm{BPH}$ resistance have been identified and mapped on rice chromosomes by marker-assisted method (Table 2 and 3 ). For more recent progress and detailed information on these BPH resistance genes/QTLs, see Jena et al. 2006, Chen et al. 2006, Jairin et al. 2007, Rahman et al. 2009, Brar et al. 2009, Myint et al. 2012.

Table 2. Recently identified and/or mapped additional major brown planthopper resistant genes

\begin{tabular}{|c|c|c|}
\hline Gene & Cllromosome & References \\
\hline Bpht3 & $6 \mathrm{~S}$ & Jairin et al. 2007 \\
\hline Bpht12(t) & $4 \mathrm{~S}$ & Yang et al. 2002, Rahuman et al. 2009 \\
\hline Bpt13(t) & $2 \mathrm{~L}$ & Liu et al. 2001 \\
\hline Bphl13(t) & $3 \mathrm{~S}$ & Renganayaki et al. 2002 \\
\hline Bptl14 (Qppt1) & $3 \mathrm{~L}$ & Yang et al. 2004 \\
\hline Bpht1S(Qppt2) & $4 \mathrm{~S}$ & Yang et al. 2004, Rahmann et al. 2009 \\
\hline Bptl $6(t)$ & 12 & Hirabayashi et al. 2004, Myint et al 2012 \\
\hline Bptl 17(t) & $4 \mathrm{~S}$ & Sun et al. 2005, Rahman et al. 2009 \\
\hline$B p h 18(t)$ & $12 \mathrm{~L}$ & Jenla et $a l .2006$, Myint et $a l .2012$ \\
\hline$B p t 19(t)$ & $3 \mathrm{~S}$ & Chen et al. 2006 \\
\hline$B p t 2 \alpha(t)$ & 4 & Rahuman et al. 2009 \\
\hline Bpt21(t) & 12 & Rahuman et al. 2009 \\
\hline Bpht22(t) & & Ram et al. 2010, Rahuman et al. 2009 \\
\hline Bpt23(t) & & Ram et al. 2010, Rahuman et al. 2009 \\
\hline Bpt2 $4(t)$ & & Deen et $\alpha$. 2010, Rahmman et $\alpha$. 2009 \\
\hline$B P H 25$ & $6 \mathrm{~S}$ & Myint et al. 2012 \\
\hline BPH26 & $12 \mathrm{~L}$ & Myint et al . 2012 \\
\hline
\end{tabular}

Table 3. List of brown planthopper resistance QTLs mapped on rice chromosomes

\begin{tabular}{l|l|l}
\hline QTLs & Chromosome & Reference \\
\hline Qbp 1 & $3 \mathrm{~L}$ & Huang et al. 2001 \\
Qbp $1($ Bph14t) & 3 & Ren et al. 2004 \\
Qbp2 $($ Bph15t) & 4 & Ren et al. 2004 \\
Qbph1 $($ Bph14 $)$ & $3 \mathrm{~L}$ & Yang et al. 2004 \\
Qbph2 $($ Bph15 $)$ & $4 \mathrm{~S}$ & Yang et al. 2004 \\
Qbph3 & 3 & Sun et al. 2005 \\
Qbph4 & 4 & Sun et al. 2005 \\
Qbph4 & $4 \mathrm{~S}$ & Liu et al. 2009 \\
Qbph6 & $6 \mathrm{~S}$ & Sun et al. 2007 \\
Qbph7 & 7 & Liu et al. 2009 \\
Qbph9 & & Liu et al. 2009 \\
Qbph10 & 10 & Sun et al. 2005 \\
\hline
\end{tabular}


Prem N. Sharma et al./Conventional and Molecular Studies .......

The pyramiding and cloning of $\mathrm{BPH}$ resistance genes/QTLs should provide the sustainable means to slow down the development of new BPH biotypes and consequently resolving the problem of $\mathrm{BPH}$ resistance breakdown in rice in Asia.

The breakdown of monogenic resistance by new BPH biotypes has been a serious threat in BPH management in rice production in Asia. For solving such a problem of resistance breakdown by the occurrence of new insect biotypes, rice varieties should be developed/ identified with durable resistance. Pyramiding of BPH resistance genes/QTLs should provide a sustainable means for developing the durable resistance against such frequently occurring new BPH biotypes (Alam \& Cohen 1998, Huang et al. 2001, Xu et al. 2002). Two approaches can be utilized for pyramiding of BPH resistance genes and QTLs. One approach is through resistance breeding by crossing among the $\mathrm{BPH}$ resistance genes/QTLs carrier rice lines/germplasms/ varieties and selecting pyramided lines by markerassisted selection. Another is cloning of BPH resistance genes/QTLs and introducing them into rice cultivars/varieties through transformation that greatly facilitates and speeds up the breeding programs to develop durable resistance against such new BPH biotypes. Several cases have been reported for pyramiding of insect resistance genes. Pyramiding of $\mathrm{BPH}$ resistance genes, Bph1 and Bph2, has been successfully achieved by marker-assisted method (Sharma et al. 2004) (Fig. 3). An estimated resistance level of the pyramided line was equivalent to Bphl but higher than $b p h 2$. Two other BPH resistance genes, $B p h 14$ and Bph15, have been reported to be pyramided, such lines showed a higher resistance than the singlegene lines (Li et al. 2006, Brar et al. 2009). Pyramiding of green rice leafhopper (GRH) resistance genes, Grh2 and $G r h 4$, also resulted in a higher level of resistance in the pyramided line than in the line carrying one of these two genes (Fujita et al. 2006, Myint et al. 2012). In the latest case, the $\mathrm{BPH}$ resistance genes, $\mathrm{BPH} 25$ and $B P H 26$, have been pyramided, which have shown a higher level of resistance (Myint et al. 2012).
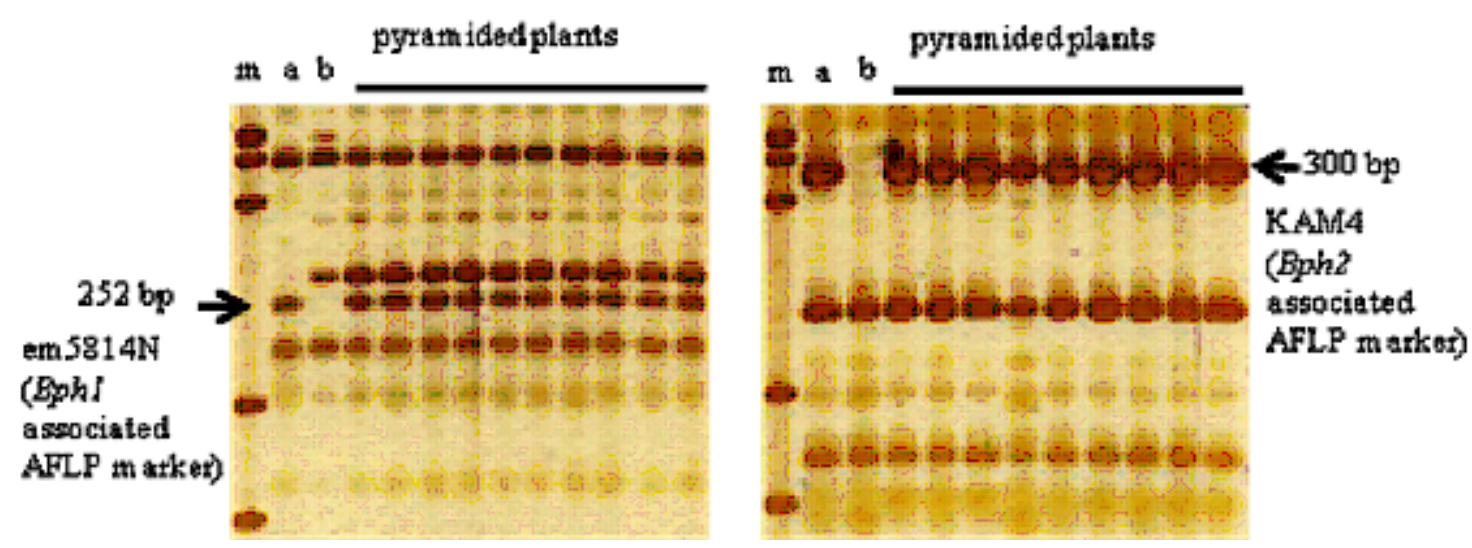

Fig. 3. Marker assisted pyramiding of BPH resistance genes Bph1 and Bph2 in rice. Both Bph1 and Bph2 associated markers are present in the pyramided plants. a \& b: resistant \& susceptible checks, respectively. m: DNA size marker (Sharma et al 2004)

Towards pyramiding of BPH resistance genes/QTLs and map-based cloning and ultimately developing the durable resistant varieties against new BPH biotypes, a variety of major genes/QTLs of BPH resistance should be identified and mapped on rice chromosomes. Studies on BPH resistance QTLs have in fact revealed that the number of resistance QTLs in the rice germplasms is very large, and suggested that the level of the quantitative resistance to BPH can be further increased by pyramiding many responsible QTLs through marker-assisted selection (Sharma et al. 2003a, $\mathrm{Xu}$ et al. 2002). As an urgent need, fine linkage maps for every major genes and major QTLs of BPH resistance have to be constructed to find the tightly linked molecular markers that can be utilized in pyramiding and map-based cloning of $\mathrm{BPH}$ resistance 
genes and QTLs. As described above, until now, fine linkage maps at least for Bph1 and Bph2 have already been achieved. These genes, however, cannot be used in resistance breeding because of their broken resistance by virulent $\mathrm{BPH}$ biotypes in most of the rice growing areas in Asia. Despite this fact, successful cloning of these genes is still necessary as a first step to understand the structure, function and expression of the natural BPH resistance genes, which should provide a fundamental knowledge for the evaluation of putative paralogues/alleles of the resistance genes that, in turn, should provide an insight into solving the serious problem of resistance breakdown by new BPH biotypes. In a cloning strategy, the PCR-based markers already obtained in these studies should be useful to screen a rice BAC (bacterial artificial chromosome) library that is constructed using the pyramid lines of Bph1 and Bph2 (Sharma et al. 2003a, Sharma et al. 2004). A contig within the introgressed region of the chromosome can be constructed in future as the next step towards the map-based cloning of these two major BPH resistance genes.

\section{Outbreaks of BPH and future strategy/ prospect of BPH resistance study in Nepal}

In Nepal, BPH was formerly a secondary pest but nowadays it has become a major pest of rice. Farmers generally use broad-spectrum insecticides as a quick, cheap and easy means of insect control. However, excessive use of chemicals has caused negative effects such as insect resurgence, destruction of natural enemies/non-targeted pests and development of pesticide resistance in insect populations including BPH. As a result, serious and frequent outbreaks of $\mathrm{BPH}$ have been reported in the past years in different parts of Nepal. The serious outbreaks of BPH occurred in Chandradangi, Jhapa in 1977 and 1978, in Sunsari and Makawanpur in 1988, in Kumroj and Kathar of Chitwan in 1996, and in various regions of Dhanusha, Mahottari, Bara, Rupandehi, Kapilvastu, Parsa, Rautahat, Sunsari, Morang, Udayapur, and Siraha districts in 1998. Damages caused by BPH outbreaks were more serious particularly in Dhanusha, Bara and Kapilvastu (Manandhar 1999, Pokhrel et al. 1998-1999). The peak infestation in most of these regions occurs in September and October causing most serious damage. The rice variety Basmati was found more susceptible whereas a local variety Valsari was found comparatively less susceptible to $\mathrm{BPH}$ in the main season (Manandhar 1999). In Chitwan district, the early season rice is often damaged with peak infestation in the later part of May (Manandhar 1999, Pokhrel et al. 1998-1999). The rice variety $\mathrm{CH}-45$ was planted mostly as the early season rice in Chitwan and the total area damaged was estimated to be 1,568 hectares with an estimated grain yield loss of 4,262 mt (Pokhrel et al. 1998-1999). Recently, the rice varieties Makawanpur-1 and Mansuli have been reported to be moderately tolerant to BPH (Paneru \& Giri 2011).

BPH has recently become more widespread in the eastern to western parts of Terai region and also in river basin areas of hilly region in Nepal. Severe form of outbreaks causing extensive damage to rice crops might occur at any time in future. Work on varietal resistance as a fundamental component of IPM needs to be initiated soon to prevent any future possible severe outbreaks of BPH. As an urgent need, the released varieties and promising lines of rice that are being regularly developed every year in the rice commodity program and rice breeding program in NARC (Nepal Agricultural Research Council) have to be screened for their resistance response to BPH. Both laboratory and field screening methods can be adopted to identify BPH resistant and susceptible rice lines. Identified promising BPH resistant varieties of rice can be released and directly recommended to farmers to grow wherever and whenever the BPH outbreaks can be predicted. To search for sources of resistance, large numbers of rice cultivars/germplasms/varieties should be collected from inside and outside the country/IRRI and they have to be screened for sources of BPH resistance which can be utilized in breeding programs to develop the BPH resistant varieties. To perform effective varietal resistance evaluation, adequate facilities need to be established for BPH bioassay such as mass rearing of $\mathrm{BPH}$ and screening for its resistance. There should also be the provisions of well-equipped laboratory and glass house facilities to strengthen the programs. Studies of BPH biotypes and differential lines of rice need to be carried on simultaneously as the prerequisites to further progress the resistance programs. Differential lines of rice in response to BPH biotypes need to be identified and maintained. BPH biotypes in relation to BPH resistance genes should also be studied, characterized and maintained. After the basic works and studies on varietal resistance are achieved, the varietal resistance programs can be further advanced with molecular marker technology. 
Prem N. Sharma et al./Conventional and Molecular Studies

National and international collaborations are required to integrate molecular marker technology in the BPH resistance works. The following are the general areas/ guidelines/long term strategy to study BPH resistance in rice by molecular marker assisted method: (i) BPH resistant lines have to be differentiated for whether they are controlled by monogenic or QTL gene/s. (ii) Monogenic resistant lines should be distinguished for whether a new gene or an already identified gene is conferring resistance on them. (iii) Chromosomal assignment and linkage mapping have to be performed for the newly identified genes. (iv) Fine linkage maps need to be constructed for major genes to identify the tightly linked molecular markers associated to these genes. (v) Pyramiding of major genes can be performed based on the tightly linked molecular markers. (vi) Map-based cloning can be proceeded for the genes that have the fine linkage maps. (vii) The structure, function, and expression can be studied for the cloned genes that can be utilized to develop transformed and pyramided lines and to study the mechanism of resistance at the molecular level for natural insect resistance genes.

$\mathrm{BPH}$ has been a serious threat in rice production in Asia. Use of durably resistant rice varieties in IPM program is the best option to control this insect. Searching and development of rice varieties with durable resistance provides a sustainable means to combat against the frequently occurring new BPH biotypes. Marker-assisted pyramiding and cloning of BPH resistance genes/QTLs are urgently required to develop durable resistance against BPH. Many resistance genes and QTLs of BPH resistance has already been identified and mapped on rice chromosomes. Construction of high resolution linkage maps for major genes and QTLs of BPH resistance is highly required to enable map based cloning and pyramiding of these genes (for more recent progress see Jena et al. 2006, Chen et al. 2006, Jairin et al. 2007, Rahman et al. 2009, Myint et al. 2012). Map-based cloning of these genes is an essential step towards understanding the structure, function and regulation of expression of these natural resistance genes, which should provide a fundamental knowledge for evaluation of putative paralogues/ alleles of the resistance genes that, in turn, should solve the serious problem of resistance breakdown by new BPH biotypes.
$\mathrm{BPH}$ is increasingly becoming a serious threat in rice production in Nepal as well. Varietal resistance works need to be initiated soon to identify and develop the $\mathrm{BPH}$ resistant rice varieties. As an urgent need, the released varieties and promising lines of rice that are being regularly developed every year in NARC have to be screened for their resistance/susceptibility response to $\mathrm{BPH}$. Identified promising $\mathrm{BPH}$ resistant rice varieties can be directly recommended to farmers as an immediate solution to prevent any future possible outbreaks of BPH in Nepal, if the outbreaks can be predicted. Simultaneously, the molecular marker technology need to be integrated in the BPH resistance works to further progress and advance the varietal resistance works in Nepal.

\section{References}

Anonymous. 1975. Brown planthopper infestation in various countries. Rice Entomol. Newsl. 3: 3.

Alam, S.N. and M.B. Cohen. 1998. Detection and analysis of QTLs for resistance to the brown planthopper, Nilaparvata lugens, in a doubled-haploid rice population. Theor. Appl. Genet. 97: 1370-1379.

Athwal, D.S., M.D. Pathak, E.H. Bacalangco and C.D. Pura. 1971. Genetics of resistance to brown planthoppers and green leafhoppers in Oryza sativa L. Crop Sci. 11: 747750.

Brar, D.S., P.S. Virk, K.K. Jena and G.S. Khush. 2009. Breeding for resistance to planthoppers in rice, 401-428 pp. In: Planthoppers: new threats to the sustainability of intensive rice production systems in Asia (Eds. K.L. Heong \& B. Hardy). International Rice Research Institute, Los Banõs, The Philippines.

Chelliah, S. and E.A. Heinrichs. 1980. Factors affecting insecticide-induced resurgence of the brown planthopper, Nilaparvata lugens on rice. Environ. Entomol. 9: 773777.

Chen, J. W., L. Wang, X.F. Pang and Q.H. Pan. 2006. Genetic analysis and fine mapping of a rice brown planthopper (Nilaparvata lugens Stal.) resistance gene bph19(t). Mol. Gen. Genomics 275: 321-329.

Choi, S.Y. 1979. Screening methods and sources of varietal resistance. In: Brown planthopper, threat to rice production in Asia. International Rice Research Institute, Los Banõs, The Philippines. 171-186 pp.

Cohen, M. B., S.N. Alam, E.B. Medina and C.C. Bernal. 1997. Brown planthopper, Nilaparvata lugens, resistance in rice cultivar IR64: mechanism and role in successful $N$. lugens management in central Luzon, Philippines. Entomol. Exp. Appl. 85: 221-229.

Deen, R., K. Ramesh, S.K. Gautam, Y.K. Rao, V.J. Lakshmi, B.C. Viraktamath, D.S. Brar and T. Ram. 2010. Identification of new gene for BPH resistance introgressed from O. rufipogon. Rice Genet. Newsl. 25:70-72. 
Feuer, R. 1976. Biotype 2 brown planthopper in the Philippines. Int. Rice Res. Newsl. 1(1): 15.

Fujita, D., K. Doi, A. Yoshimura and H. Yasui. 2006. Molecular mapping of a novel gene, Grh5, coferring resistance to green rice leafhopper (Nephotettix cincticeps Uhler) in rice, Oryza sativa L. Theor. Appl. Genet. 113: 567-573.

Georghiou, G.P. 1986. The magnitude of the resistance problems, pesticide resistance, strategies and tactics for management. National Academy Press, Washington DC, 14-43 pp.

Heinrichs, E.A., G.B. Aquino, S. Chelliah, S.L. Valencia and W.H. Reissig. 1982. Resurgence of Nilaparvata lugens (Stål) populations as influenced by method and timing of insecticide applications in lowland rice. Environ. Entomol. 11: 78-84.

Hirabayashi, H. and T. Ogawa. 1995. RFLP mapping of Bph1 (Brown planthopper resistance gene) in rice. Japan. J. Breed. 45: 369-371.

Hirabayashi, H., E.R. Angeles, R. Kaji, T. Ogawa, D.S. Brar and G.S. Khush. 1998. Identification of brown planthopper resistance gene derived from $O$. officinalis using molecular markers in rice (abstract in Japanese). Breed. Sci. 48 (1): 82.

Hirabayashi, H., R. Kaji, E.R. Angeles, T. Ogawa, D.S. Brar and G.S. Khush. 1999. RFLP analysis of a new gene for resistance to brown planthopper derived from $O$. officinalison rice chromosome 4 (abstract in Japanese). Breed. Sci. 49 (1): 48.

Huffaker, C.B. and R.F. Smith. 1980. Rationale, organization and development of a national integrated pest management project. In: New technology of pest control (Ed. C.B. Huffaker) John Wiley and Sons, New York. pp.1-24.

Huang, Z., G. He, L. Shu, X. Li and Q. Zhang. 2001. Identification and mapping of two brown planthopper resistance genes in rice. Theor. Appl. Genet. 102: 929-934.

Ikeda, R. and C. Kaneda. 1983. Trisomic analysis of the Bphl for resistance to the brown planthopper, Nilaparvata lugens Stål, in rice. Japan. J. Breed. 33: 40-44.

Ikeda, R. 1985. Studies on the inheritance of resistance to the rice brown planthopper (Nilaparvata lugens Stål) and the breeding of resistant rice cultivars. Bull. Natl. Agric. Res. Cent. 3: 1-54.

Ishii, T., D.S. Brar, D.S. Multani and G.S. Khush. 1994. Molecular tagging of genes for brown planthopper resistance and earliness introgressed from Oryza australiensis into cultivated rice, O. sativa. Genome. 37: 217-221.

IRRI. 1982. A brown planthopper population that feed on IR 36. Ann. Rep. for 1981: 56-58.

Jairin, J., K. Phengrat, S. Teangdeerith, A. Vanavichit and T. Toojinda T. 2007. Mapping of a broad-spectrum brown planthopper resistance gene, $B p h 3$, on rice chromosome 6. Mol. Breeding 19: 35-44.

Jena, K.K., J.U. Jeung, J.H. Lee, H.C. Choi and D.S. Brar. 2006. High-resolution mapping of a new brown planthopper (BPH) resistance gene, Bph18(t), and marker-assisted selection for BPH resistance in rice (Oryza sativa L.). Theor. Appl. Genet. 112: 288-297.

Jeon, Y., S. Ahn, H. Ahn, T. Hahn and H. Moon. 1999. Identification of a RAPD marker linked to a brown planthopper resistance gene in rice. Euphytica 107: 23-28. Printed in the Netherlands.

Jena, K.K. and G.S. Khush. 1990. Introgression of genes from Oryza officinalis Well x Watt to cultivated rice, O. sativa L. Theor. Appl. Genet. 80: 737-745.

Kaneda, C. and R. Kisimoto. 1979. Status of varietal resistance to the brown planthopper in Japan. In: Brown Planthopper Threat to Rice Production in Asia. International Rice Research Institute, Los Banõs, The Philippines. pp.209-218.

Kabir, M.A. and G.S. Khush. 1988. Genetic analysis of resistance of brown planthopper in rice, Oryza sativa L. Plant Breed. 100: 54-58.

Kawaguchi, M., K. Murata, T. Ishii, S. Takumi, N. Mori and C. Nakamura. 2001. Assignment of a brown plant hopper (Nilaparvata lugens Stål) resistance gene bph4 to the rice chromosome 6. Jpn. J. Breed Sci. 51: 13-18.

Khush, G.S., A.N.M. Rezaul Karim and E.R. Angeles. 1985. Genetics of resistance of rice cultivar ARC10550 to Bangladesh brown planthopper biotype. J. Genet. 64: 121-125.

Khush, G. S. 1989. Multiple disease and insect resistance for increased yield stability in rice. In: Progress in Irrigated Rice Research. International Rice Research Institute, Manila, The Philippines. pp.79-92.

Lakshminarayana, A. and G.S. Khush. 1977. New genes for resistance to brown planthopper in rice. Crop Sci. 17: 96-100.

Li, J.B., M.Y. Xia, Q. HX, G.C. He, B.L. Wan and Z.P. Zha. 2006. Marker-assisted selection for brown planthopper (Nilaparvata lugens Stål) resistance genes Bphl4 and Bph15 in rice. Sci. Agric. Sin. 39: 21322137.

Ling, K.C., E.R. Tiongco and V.M. Aguiero. 1978. Rice ragged stunt, a new virus disease. Plant Dis. Rep. 62: 701-705.

Liu, G.Q., H.H. Yan, Q. Fu, Q. Qian, Z.T. Zhang, W.X. Zhai and L.H. Zhu. 2001. Mapping of a new gene for brown planthopper resistance in cultivated rice introgressed from Oryza eichingeri. Chin. Sci. Bull. 46:1459-1462.

Liu, Y., C. Su, L. Jiang, J. He, H. Wu, C. Peng and J. Wan J. 2009. The distribution and identification of brown planthopper resistance genes in rice. Hereditas 146 : 67-73.

Manandhar, D.N. 1999. Rice and brown planthopper: Areview. NARC/ CIMMIT, Nepal. 
Prem N. Sharma et al./Conventional and Molecular Studies .......

Mano, Y., S. Kawasaki, F. Takaiwa and T. Komatsuda. 2001. Construction of a genetic map of barley (Hordeum vulgare L.) cross 'Azumamugi' x 'Kanto Nakate Gold' using a simple and efficient amplified fragment-length polymorphism system. Genome. 44: 284-292.

Michelmore, R. W., I. Paran and R.V. Kesseli. 1991. Identifications of markers linked to disease resistance genes by bulked segregant analysis: A rapid method to detect markers in specific genomic regions by using segregating populations. Proc. Natl. Acad. Sci. 88: 9828-9832.

Murata, K., C. Nakamura, N. Mori and C. Kaneda. 1997. Tagging and mapping of brown planthopper resistance genes in rice. In: Proceed. $5^{\text {th }}$ Internat. Symp. on Rice Mol. Biol. (Ed. J.C. Su), Yi-Hsein Pub. Co. Ltd., Taipei, Taiwan, pp.217-231.

Murata, K., M. Fujiwara, C. Kaneda, S. Takumi, N. Mori and C. Nakamura. 1998. RFLP mapping of a brown planthopper (Nilaparvata lugens Stål) resistance gene bph2 of indica rice introgressed into a japonica breeding line 'Norin-PL4'. Genes Genet. Syst. 73: 359-364.

Murai, H., Z. Hashimoto, P.N. Sharma, T. Shimizu, K. Murata, S. Takumi, N. Mori, S. Kawasaki and C. Nakamura. 2001. Construction of a high-resolution linkage map of a rice brown planthopper (Nilaparvata lugens Stål) resistance gene bph2. Theor. Appl. Genet. 103: 526-532.

Myint, K.K.M., D. Fujita, M. Matsumura, T. Sonoda, A. Yoshimura and H. Yasui. 2012. Mapping and pyramiding of two major genes for resistance to the brown planthopper (Nilaparvata lugens [Stål] in the rice cultivar ADR52. Theor. Appl. Genet. 124: 495-504.

Nemoto, H., R. Ikeda, and C. Kaneda. 1989. New genes for resistance to brown planthopper, Nilaparvata lugens Stål., in rice. Jpn. J. Breed. 39: 23-28.

Paneru, R.B. and Y.P. Giri. 2011. Management of economically important agricultural and household pests of Nepal. NARC, Entomology Division, Khumaltar, Lalitpur, Nepal, 136 pp.

Pokhrel, S., P.N. Sharma and F.P. Neupane. 1998-1999. Incidence and control of the rice plant-hoppers in Chitwan, Nepal. J. Inst. Agric. Anim. Sci. 19-20: 55- 64.

Rahman, M.L., W. Jiang, S.H. Chu, Y. Qiao, T.H. Ham, M.O. Woo, J. Lee, M.S. Khanam, J.H. Chin, J.U. Jeung, D.S. Brar, K.K. Jena and H.J. Koh. 2009. High-resolution mapping of two rice brown planthopper resistance genes, $B p h 20(t)$ and Bph21(t), originating from Oryza minuta. Theor. Appl. Genet. 119: 1237-1246.

Ram, T., R. Deen, S.K. Gautam, K. Ramesk, Y.K. Rao and D.S. Brar. 2010. Identification of new genes for brown planthopper resistance in rice introgressed from $O$. glaberrima and O. minuta. Rice Genet. Newsl. 25:67-69.
Rengawanayaki, K., A.K. Fritz, S. Sadasivam, S. Pammi, S.E. Harrington, S.R. McCouch, S.M. Kumar and A.S. Reddy. 2002. Mapping and progress toward map-based cloning of brown planthopper biotype-4 resistance gene introgressed from Oryza officinalis into cultivated rice, O. sativa. Crop Sci. 42: 2112-2117.

Ren, X., X. Wang, H. Yuan, Q. Weng, L. Zhu and G. He. 2004. Mapping quantitative trait loci and expressed sequence tags related to brown planthopper resistance in rice. Plant Breed. 123: 342-348.

Rivera, C.T., S.H. Ou and T.T. Iida. 1966. Grassy stunt disease of rice and its transmission by the brown planthopper, Nilaparvata lugens Stål. Plant Dis. Rep. 50: 453-456.

Salim, M. and E.A. Heinrichs. 1987. Insecticide-induced changes in the level of resistance to rice cultivars to the white-backed planthopper, Sogatella furcifera (Horvath) (Homoptera: Delphacidae). Crop Prot. 6: 28-32.

Sharma, P.N., A. Tori, S. Takumi, N. Mori and C. Nakamura. 2003a. Towards molecular cloning of resistance genes against brown planthopper (Nilaparvata lugens Stål) in rice: a case study of natural insect resistance genes. Trends in Entomology 3: 87-96.

Sharma, P.N., Y. Ketipearachchi, K. Murata, A. Torii, S. Takumi, N. Mori and C. Nakamura. 2003b. RFLP/AFLP mapping of a brown planthopper (Nilaparvata lugens Stål) resistance gene Bphl in rice. Euphytica 129: 109-117.

Sharma, P.N., A. Torii, S. Takumi, N. Mori, and C. Nakamura. 2004. Marker-assisted pyramiding of brown planthopper (Nilaparvata lugens Stål) resistance genes Bph1 and Bph2 on rice chromosome 12. Hereditas 140: 1-9.

Sogawa, K. and C.H. Cheng. 1979. Economic thresholds, nature of damage and losses caused by the brown planthopper. In: Brown planthopper: Threat to rice production in Asia. IRRI, Los Banos, Philippines. Pp.125-144.

Stapley, J.H. 1975. The problem of the brown plant hopper (Nilaparvata lugens) on rice in Solomon Islands. Rice Entomol. Newsl. 2: 37.

Sun, L., C. Su, C. Wang, H. Zhai, J. Wan. 2005. Mapping of a major resistance gene to the brown planthopper in the rice cultivar Rathu Heenati. Breed Sci 55:391-396.

Sun, L., Y. Liu, L. Jiang, C. Su, G. Wang, H. Zhai, J. wan. 2007. Identification of quantitative trait loci associated with resistance to brown planthopper in the indica rice cultivar Col.5 Thailand. Hereditas: 144: 48-52.

Xu, X. F., H.W. Mei, L. J. Luo, X.N. Cheng and Z. K. Li. 2002. RFLP-facilitated investigations of the quantitative resistance of rice to brown planthopper (Nilaparvata lugens). Theor. Appl. Genet.104: 248-253.

Yang, H., A. You, Z. Yang, F. Zhang, R. He, L. Zu, G. He. 2004. High resolution genetic mapping at the Bph15 locus for brown planthopper resistance in rice (Oryza sativa L.). Theor. Appl. Genet. 110:182-191. 
Nepal Journal of Science and Technology Vol. 15, No.1 (2014) 145-156 